\title{
Acute eosinophilic pneumonia associated with elevated NKT cell response in COVID-19 patients
}

\section{Dong-Min Kim ( $\nabla$ drongkim@chosun.ac.kr)}

Department of Internal Medicine, Chosun University College of Medicine Jun-Won Seo

Department of Internal Medicine, Chosun University College of Medicine

\section{Yuri Kim}

Department of Microbiology and Immunology, Seoul National University College of Medicine and Division of Infectious Diseases, Department of Internal Medicine, Chungnam National University School of Medicine

\section{Uni Park}

Department of Microbiology and Immunology, Department of Biomedical Sciences, Seoul National University College of Medicine

\section{$\mathrm{Na}$-Young $\mathrm{Ha}$}

Department of Microbiology and Immunology, Seoul National University College of Medicine and Division of Infectious Diseases, Department of Internal Medicine, Chungnam National University School of Medicine

\section{Hyoree Park}

Department of Microbiology and Immunology, Department of Biomedical Sciences, Seoul National University College of Medicine

\section{Na Ra Yun}

Department of Internal Medicine, Chosun University College of Medicine

\section{Da Young Kim}

Department of Internal Medicine, Chosun University College of Medicine

\section{Sung Ho Yoon}

Department of Internal Medicine, Chosun University College of Medicine

\section{Yong Sub Na}

Department of Internal Medicine, Chosun University College of Medicine

\section{Do Sik Moon}

Department

\section{Sung-Chul Lim}

Department of Pathology, Chosun University College of Medicine

\section{Choon-Mee Kim}

Premedical Science, Chosun University College of Medicine 
Department of Microbiology and Immunology, Department of Biomedical Sciences, Seoul National University College of Medicine

\section{Jun-Gu Kang}

Department of Microbiology and Immunology, Seoul National University College of Medicine and Division of Infectious Diseases, Department of Internal Medicine, Chungnam National University School of Medicine

\section{Yeon-Sook Kim}

Division of Infectious Diseases, Department of Internal Medicine, Chungnam National University School of Medicine

\section{Nam-Hyuk Cho ( $\square$ chonh@snu.ac.kr)}

Department of Microbiology and Immunology, Department of Biomedical Sciences, Seoul National University College of Medicine and Institute of Endemic Disease, Seoul National University Medical Research Center and Bundang Hospital

\section{Research Article}

Keywords: COVID-19, SARS-CoV-2, eosinophilia, pneumonia, and bronchoalveolar lavage

Posted Date: April 17th, 2020

DOI: https://doi.org/10.21203/rs.3.rs-23607/v1

License: (a) (1) This work is licensed under a Creative Commons Attribution 4.0 International License. Read Full License 


\section{Abstract}

Coronavirus disease 2019 (COVID-19) is caused by severe acute respiratory syndrome coronavirus-2 (SARS-CoV-2) ${ }^{1}$ and often results in fatal pneumonia. ${ }^{2,3}$ Despite the worldwide effect of the COVID-19 pandemic, the underlying mechanisms of the fatal viral pneumonia remain elusive. Here, we investigated respiratory specimens, including broncholoalveolar lavage fluids (BALFs) and bloods collected from three confirmed cases of COVID-19 patients with varying degrees of disease severity. Surprisingly, over $35 \%$ of cells from BALFs obtained from two pneumonic patients was comprised of eosinophils, while $20 \%$ was comprised of lymphocytes. Cytological analysis of sputa and tracheal aspirates from all three patients also revealed that more than $90 \%$ of total cells were eosinophils. Infiltration of $\mathrm{CD} 16^{+} / \mathrm{CD} 24^{+}$ polymorphonuclear cells into lungs, together with elevated NKT cells in BALFs and peripheral blood samples, in patients with severe pneumonia was confirmed by flow cytometry. Moreover, rapid and profound IgE responses against the $\mathrm{N}$ protein of SARS-CoV-2 was only detected in plasma from a patient suffering from more severe and prolonged pneumonia. A significant reduction in oxygen demand with improved chest imaging was observed in two severe COVID-19 patients after treatment with a steroid, methylprednisolone. The present study provides evidence that acute eosinophilic pneumonia ${ }^{4}$ is associated with COVID-19.

\section{Introduction}

In December 2019, a novel coronavirus was found to be the cause of the pneumonia cluster emerging from Wuhan, China.1 Consequently, the World Health Organization (WHO) named this disease as coronavirus disease 2019 (COVID-19) in February 2020. The virus causing COVID-19 was identified as severe acute respiratory syndrome coronavirus-2 (SARS-CoV-2). 5 Currently, SARS-CoV-2 has been rapidly spreading worldwide via infected travelers and has caused more than 1.9 million infections with an average mortality rate of approximately $6 \%$, but ranging from less than $1 \%$ to more than $10 \%$, depending on the affected country (https://covid19.who.int). The primary cause of disease fatality is severe pneumonia, resulting in acute respiratory syndrome (ARDS). 3 Around $80 \%$ of confirmed cases are asymptomatic or have mild symptoms, including fever, cough, sore throat, and myalgia, whereas the rest often develop severe pneumonia requiring supplemental oxygen therapy.2,6 The most common finding of radiological imaging is bilateral, ground-glass opacity in the periphery of the lungs. 2 Nevertheless, it is also notable that pneumonia is detected radiologically in the majority $(\sim 80 \%)$ of symptomatic patients as early as the first day of illness onset, and was even identified in patients who did not have any symptoms of the lower respiratory tract infection, such as cough, sputum, chest pain, or dyspnea. 3 Although they had radiological pneumonia, they did not feel unwell and were able to carry on their daily activities as usual ("walking pneumonia").3 The mechanisms underlying this various degree of pneumonia severity observed in COVID-19 patients remain elusive.

In order to characterize the cause of viral pneumonia in COVID-19 patients, we analyzed the immunological features of respiratory and blood specimens collected from three confirmed patients who have various degrees of clinical symptoms: one with mild symptoms without pneumonia and two with 
severe pneumonia. We systematically analyzed plasma, leukocytes in blood and bronchoalveolar lavage fluids (BALFs), sputa, and tracheal aspirates to characterize immunological responses upon viral infection. We also compared systemic and local responses in circulating blood and inflamed lungs using the clinical specimens. To our surprise, this extensive analysis revealed that COVID-19 is consistently associated with acute eosinophilic infiltration into infected lungs in all three patients. In addition, elevated NKT cell responses were observed in the patients regardless of disease severity. These results indicate that SARS-CoV-2 infection may induce specific innate immune responses, including NKT cells and eosinophils, in respiratory tract and subsequent lung inflammation to result in COVID-19 associated pneumonia.

\section{Results And Discussion}

Clinical courses and baseline characteristics of the three patients included in this study is are summarized in Table 1 and Fig. 1a. The detailed epidemiological information and clinical features are described in the Methods section. P1 and P2 had acute and severe pneumonia requiring mechanical ventilation. $\mathrm{P} 2$ suffered from more prolonged and severe type of pneumonia than $\mathrm{P} 1$, as evidenced by radiological chest imaging (Fig. 1b). P3 had only mild symptoms without pneumonia.

Initially, we analyzed BALFs and sputa collected from the three patients in order to identify immune cell types infiltrating into infected lungs. BALF and sputa cytology analyses using H\&E staining showed that the majority of inflammatory cells were composed of eosinophils, and a few macrophages and lymphocytes (Table 1 and Fig. 1c). BALF analysis of two COVID-19 patients with severe pneumonia (P1 and P2) revealed that lymphocytes accounted for $20 \%$ and eosinophils accounted for more than $35 \%$ of inflammatory cells, indicating acute eosinophilic pneumonia. ${ }^{4}$ The cytological analysis of sputa and tracheal aspirates collected from all three patients revealed that more than $90 \%$ of the cells were eosinophils (Table 1 and Fig. 1c). It is notable that a sputum sample collected at day 3 after symptom onset in P3, who did not develop pneumonia, but had mild respiratory symptoms, also showed ample eosinophils. In addition, a sputa sample obtained from P2, who rapidly developed viral pneumonia, at day 2 after symptom onset (one day after pneumonia presentation), also contained numerous eosinophils, in addition to a few other immune cells such as lymphocytes and macrophages (Table 1 and Fig. 1c). Therefore, eosinophilic infiltration into infected lungs might be rapid and universal in COVID-19 patients if they are symptomatic, regardless of the severity of pneumonia.

In order to confirm the infiltration of eosinophils into pneumonic lungs, BALFs collected from P1 at 12 days (acute phase) and 19 days (convalescent phase) after symptom onset were applied for flow cytometric analysis. Peripheral blood collected from P2 at 12 days (acute phase) and P1 at 19 days (convalescent phase) after symptom onset were simultaneously used for flow cytometry of leukocytes. We were able to identify relative proportions and kinetic changes of $\mathrm{CD}_{14}{ }^{+}$monocytes/macrophages, $\mathrm{CD}^{+} \mathrm{T}$ cells, $\mathrm{CD} 20^{+} \mathrm{B}$ cells, and $\mathrm{CD} 16^{+} / \mathrm{CD} 24^{+}$polymorphonuclear cells (PMNs), including eosinophils, among blood leukocytes and BALFs (Fig. 2). Although we used blood samples from two different patients for acute phase analyses, general trends in changes of relative proportions of the specific immune cell 
subtypes could be drawn by comparisons with blood leukocyte samples from a healthy volunteer as a control. As we observed in cytological analysis using BALFs, relative proportion of PMNs among leukocyte populations (after excluding macrophages, $T$ cells, and B cells) were elevated at acute phase $(89.6 \%)$, then slightly reduced at convalescent phase (67.2\%). Consistent with results of cytological analysis using H\&E staining, the reduced expression levels of surface CD24 molecules, ${ }^{7}$ when compared to those in a healthy control primarily containing neutrophils, from flow cytometry support that PMNs in BALFs are mainly composed eosinophils. Monocytes in peripheral blood were increased at acute phase but declined at convalescent phase. However, their proportion among total leukocytes were downregulated during acute phase but upregulated during convalescent phase in BALFs. B cells were barely detected in BALFs at acute phase but increased at convalescent phase. Selective and enhanced influx of B cells and T cells during convalescent phase might be associated with resolution of lung inflammation and play a major role in eradication of SARS-CoV-2 from infected lungs. Interestingly, the main proportion of T cells in BALFs during acute phase showed intermediate levels of surface CD3 molecules when compared to those of blood T cells, suggesting that other types of CD $3^{+}$lymphocytes may play a role in the lungs. Indeed, when we assessed the surface expression of other cellular markers, including CD24, CD16, and HLA-DM, we found that the majority of $\mathrm{CD}^{+}{ }^{+} \mathrm{T}$ cells in BALFs at acute phase were $\mathrm{CD} 16^{+} / \mathrm{CD} 24^{+} / \mathrm{HLA}^{-D M}{ }^{-}$cells, suggesting a predominantly NKT cell population (the last raw of Fig. 2 and Supplementary Fig. 2a) ${ }^{8,9}$ Elevation of NKT cells expressing both CD3 and CD56 in the patient's blood at acute phase was further confirmed by additional flow cytometric analysis using PBMCs (Fig. 3a and $b$ ). The relative levels of $\mathrm{CD}^{+} / \mathrm{CD}^{2} 6^{+}$NKT cells in the patients' blood lymphocytes at acute phase $(5.8 \pm 1.3 \%$, mean \pm S.D.) was 6.4 and 2.5 times higher than those of healthy controls $(0.9 \pm 0.8 \%)$ and patients' blood lymphocytes at convalescent phase $(2.3 \pm 1.3 \%)$, respectively. In addition, $\mathrm{CD}^{+} / \mathrm{CD} 1 \mathrm{~d}-$ tetramer ${ }^{+}$NKT $(\mathrm{iNKT})^{10}$ cells at acute phase $(1.3 \pm 0.4)$ were also significantly elevated by 4.3 and 1.6 times when compared to those of healthy controls $(0.3 \pm 0.1)$ and convalescent phase $(0.8 \pm 0.1)$, respectively. However, the levels of $\mathrm{CD}^{-} / \mathrm{CD} 56^{+} \mathrm{NK}$ cells in peripheral bloods seem to be changed variably depending on individual cases and did not significantly associated with the degree of disease severity and disease progression in the COVID-19 patients (Fig. 3). The quantitative changes in NKT cell population were consistently observed in all three patients, suggesting that upregulation of NKT cell population might be a common phenotype initiated upon SARS-CoV-2 infection regardless of disease severity. Furthermore, the relative frequency of NKT cells among lymphocytes decreased during the convalescent phase, suggesting their kinetic changes might be correlated with disease progression. In contrast, the relative proportion of $\mathrm{CD}^{+} \mathrm{T}$ cells, including $\mathrm{CD} 4^{+}$helper $\mathrm{T}$ cells and $\mathrm{CD} 8^{+}$cytotoxic $\mathrm{T}$ cells, and $\mathrm{CD} 2 \mathrm{O}^{+} \mathrm{B}$ cells were highly variable among the patients, but less degree in P3 with milder disease (Supplementary Fig. S2b). Nevertheless, the relative proportion of each subtype of blood leukocytes, as assessed by flow cytometry and complete blood count (CBC, Supplementary Fig. S3), did not correctly reflect the lung-infiltrating leukocytes, especially eosinophils and lymphocytes, revealed by direct analysis of BALFs from the patients. Therefore, we need to carefully monitor the immune leukocytes present in respiratory secretions in order to define the leukocyte population responsible for the acute lung inflammation often observed in COVID-19 patients. 
Significant elevation and infiltration of NKT cells and eosinophils into lungs of COVID-19 patients during the acute phase of infection indicates that these innate immune cells might be initial mediators of pulmonary inflammation, defining acute eosinophilic pneumonia. ${ }^{4}$ This type of immunopathological response appears to commonly occur in symptomatic COVID-19 patients regardless of disease severity, since a similar degree of eosinophilic infiltration into lungs and systemic elevation of NKT cells was also observed in P3, who did not have significant pneumonia. The specific role of NKT cells in eosinophilic pneumonia has been previously reported in a mice model of allergic asthma. ${ }^{11}$ Moreover, it has been shown that $\mathrm{CD}^{+} \mathrm{CD} 16 / 56^{+} \mathrm{NKT}$ cell levels are positively correlated with eosinophil percentage in BALF samples from human eosinophilic pneumonia patients. ${ }^{12}$ The association of elevated NKT cells with the pathogenesis of acute eosinophilic pneumonia is poorly understood, but likely depends on the underlying cause of airway epithelial injury caused by SARS-CoV-2 infection in case of COVID-19. Subsequently, eosinophilic infiltration and degranulation appear to mediate lung inflammation and associated clinical manifestations. ${ }^{4}$ Although peripheral blood eosinophilia at initial presentation may indicate acute eosinophilic pneumonia, it may be absent or delayed, ${ }^{4}$ as we and others observed in the COVID-19 patients (see CBC results in Supplementary Fig. S3). ${ }^{13}$

While we still do not know why some patients develop very severe pneumonia while a majority only have mild symptoms or "walking pneumonia," both might be attributable to eosinophilic infiltration into infected lungs, as seen in P3 of our current study. Therefore, we further investigated several humoral indicators potentially associated with disease severity and compared results with the kinetic changes in viral loads of respiratory secretions (Fig. 4). The kinetics of viral loads in upper (nasopharyngeal and throat swabs) and lower (sputa) respiratory tracts were similar in all three patients and these were not significantly different between severe (P1 and P2) and mild (P3) cases (Fig. 4a), although there was more sustained viral shedding in severe cases, consistent with a recent report. ${ }^{14}$ The levels of C-reactive proteins (CRP) were elevated in the severe patients during the second week after symptom onset, whereas it was not detected in a mild case, P3, suggesting CRP as a potential indicator of severe disease (Fig. 4b). ${ }^{15}$ We could also observe higher and more prolonged elevation of complements, C3a and C5a, ${ }^{16}$ in plasma of severe patients suffering from pneumonia, but this did not correlate well with the degree and duration of prolonged pneumonia as quantified by radiographical score of chest images (Fig. 1b). Since eosinophilic inflammation in lungs is associated with Th2-polarized immune responses, ${ }^{4}$ we next assessed viral antigen-specific antibody responses, with a focus on specific isotypes. We speculated that specific immune responses against the SARS-CoV-2 antigen is orchestrated by a Th2-biased immune environment, which can drive preferential isotype switching of specific antibodies in B cells toward specific subclasses, such as IgE. ${ }^{17}$ Rapid and remarkable elevation of anti-N protein antibodies, including $\lg \mathrm{G} 1$, IgG3, IgA, and IgE isotypes (IgG2 and IgG4 was not detected) was observed in plasma from P2 with prolonged severe pneumonia during the first week after symptom onset, whereas antibody responses were delayed and weaker in P1 and even barely observed in P3, indicating that the timing and degree of these isotype specific antibodies against viral $\mathrm{N}$ protein is correlated with the severity of COVID-19 pneumonia. It is noteworthy that an antigen-specific IgE response was observed only in P2 who had the 
highest morbidity. This suggests that antigen-specific lgE response could be a hallmark of severe and prolonged pneumonia that is potentiated by initial eosinophilic infiltration into infected lungs. Given that, like other eosinophilic respiratory diseases such as asthma, the early stage of COVID-19 immunologic pathogenesis involves Th2-biased immune environment in the respiratory system ${ }^{18}$ and both are potentially associated with genetic background, ${ }^{19}$ ongoing epidemiological studies of COVID-19 (incidence and/or fatality rate) could result in correlations with global asthma incidence (Fig. 4e).

Acute eosinophilic pneumonia was first reported in 1989 and is diagnosed when eosinophils exceed $25 \%$ in a BAL differential count without other causes for BAL fluid eosinophilia such as asthma or atopic disease, and medications. ${ }^{20}$ Acute eosinophilic pneumonia can occur as a result of various drug treatments and infections, such as HIV, parasitic, and fungal infections. In addition, cigarette smoking is specifically associated with an increased incidence of acute eosinophilic pneumonia, although the underlying mechanisms still remain elusive. ${ }^{4}$ Here, we showed that systemic elevation of NKT cells and their infiltration into infected lungs might be associated with the eosinophilic lung inflammation in COVID19 patients. This unique initial response could be due to epithelial damages caused by SARS-CoV-2 infection. In addition, we also observed rapid and strong IgE responses, as well as other antibody isotypes, against a viral antigen solely in a patient who suffered from more prolonged and severe pneumonia. This suggests that even antigen-specific adaptive immunity might be also affected by the Th2-biased eosinophilic responses during the acute phase, especially in more severe cases of COVID-19. It is imperative that the underlying mechanisms of the biased innate responses, as well as causative cascade toward Th2-biased adaptive immunity resulting in fatal pneumonia are characterized in following studies.

To date, there is no effective treatment for patients with COVID-19. In a lopinavir/ritonavir clinical trial among patients with severe COVID-19, there was no significant difference observed between the treatment group and those that received standard care. ${ }^{21}$ Two randomized control trials with hydroxychloroquine and three trials of combination treatment with hydroxychroroquine plus azithromycin failed to demonstrate a beneficial effect (https://www.idsociety.org/). The administration of steroids in patients infected with other coronaviruses, such as MERS or SARS coronavirus, resulted in delayed viral clearance with other short- and long-term side effects. ${ }^{22}$ WHO and CDC do not recommend the treatment of glucocorticoids in COVID-19 positive patients unless otherwise indicated, such as exacerbation of chronic obstructive pulmonary disease (https://www.cdc.gov/coronavirus/2019-ncov/). IDSA guideline panel also do not suggest the use of corticosteroids (https://www.idsociety.org/). However, systemic glucocorticoid therapy is generally considered to be the main treatment of acute eosinophilic pneumonia. ${ }^{4}$ In our study, methylprednisolone was administered to patients with rapid progressive pneumonia (P1 and P2) and then, their oxygen demand substantially decreased with improved X-ray findings. Such improvement was suspected to be the result of steroid administration. Moreover, an analysis of 201 COVID-19 positive patients from Wuhan indicated that the risk of mortality was reduced in ARDS patients with the administration of methylprednisolone. ${ }^{23}$ The efficacy of steroid treatment in decreasing mortality risk suggests that COVID-19 associated pneumonia is, in fact, acute eosinophilic 
pneumonia. Therefore, future studies on possible therapies for acute eosinophilic pneumonia in COVID-19 patients need to be conducted on a broader range, targeting eosinophilia and NKT cell response in pulmonary tract. ${ }^{24-26}$ In addition, rapid and strong elevation of anti-viral antibodies, including IgE , as we observed in a severe COVID-19 patient, needs to be carefully considered for plasma therapy (i.e. potential possibility of type I hypersensitivity). ${ }^{27}$

In conclusion, cytological and flow cytometric analysis of respiratory secretions confirmed pneumonic eosinophilia in three COVID-19-positive cases. The current study demonstrates that acute eosinophilic pneumonia with elevated NKT cells is associated with COVID-19. These observations require further confirmation with extensive research of the pathogenesis of eosinophilic pneumonia in COVID-19 patients.

\section{Methods}

\section{Ethnical statement}

The current research was approved by the institutional review boards of Chosun University Hospital (IRB no.: 2020-02-011), Chungnam National University Hospital (IRB no.: CNUH2017-12-004), and Seoul National University Hospital (IRB no.: C-1509-103-705). This study was performed in accordance with the ethical standards laid down in the 1964 declaration of Helsinki and all subsequent revisions. This study was conducted with informed consent from patients or their legal guardians.

\section{The patients}

General information on the clinical courses and baseline characteristics of the three patients included in this study are summarized in Table 1 and Fig. 1. The epidemiological and clinical features of the three patients are as follows:

A 79-year-old male (P1) developed fever and headache after contact with his daughter who had confirmed COVID-19. Reverse transcriptase-polymerase chain reaction (RT-PCR) of SARS-CoV-2 genes was performed on the first day after symptom onset, and he was also confirmed to be COVID-19 positive. On day 5 after symptom onset, he was admitted to a local hospital where they administered lopinavir/ritonavir and empiric antibiotics (ceftriaxone). On day 9, he was transferred to Chosun University Hospital due to a gradual increase in oxygen demand. At the time of his transfer, he had a high fever (body temperature: $38.9^{\circ} \mathrm{C}$ ) and developed acute respiratory distress syndrome (ARDS); thus mechanical ventilation was started. Lopinavir/ritonavir treatment was continued for a total of 11 days until day 15 after symptom onset. On day 12, bronchoscopy with BAL was performed. On day 17, corticosteroid (methylprednisolone $1 \mathrm{mg} / \mathrm{kg}$ per day) was started. A follow-up chest X-ray was completed at 2 days after corticosteroid administration (Fig. 1b), which showed significant improvement of pneumonic infiltration of both lung fields and a decrease in $\mathrm{O}_{2}$ demand $\left(\mathrm{FiO}_{2}\right.$ from 0.8 to 0.4$)$. He was thereafter stable with continuous mechanical ventilation until day 26 after symptom onset. 
A 79-year-old female (P2) was confirmed as COVID-19 positive while asymptomatic and began to selfquarantine at home. 2 days later, she showed shortness of breath and was admitted to a hospital. She had dyspnea with gradual aggravation and showed an increase in $\mathrm{O}_{2}$ demand after the first day of hospitalization, so she was transferred to Chosun University Hospital. She had a fever (body temperature: $38.3^{\circ} \mathrm{C}$ ) and showed pneumonic infiltration of both lung fields in the chest X-ray at the time of assessment (Fig. 1b). Mechanical ventilation was started because of persistent hypoxemia with $\mathrm{O}_{2}$ saturation of $85 \%$ despite the application of nasal high flow therapy. Lopinavir/ritonavir was administered for a total of 10 days from the first day of symptom onset, but there was no improvement in pneumonia. Therefore, bronchoscopy with BAL was performed on day 13 . On day 14 , corticosteroid (methylprednisolone $1 \mathrm{mg} / \mathrm{kg}$ per day) was started and on day 16 , there were signs of improvement of pneumonia on the follow-up chest X-ray (Fig. 1 b). $\mathrm{O}_{2}$ demand also decreased $\left(\mathrm{FiO}_{2}\right.$ from 0.7 to 0.4 ), and weaning from mechanical ventilation was successful on day 22. After re-examination, she was confirmed to be SARS-CoV-2 negative at least twice in 24-hour intervals on day 25 and day 27 after symptom onset, and she was ultimately stable with T-piece $\mathrm{O}_{2} 3 \mathrm{~L}$.

A 36-year-old male (P3) with no underlying disease condition. Although asymptomatic, he visited the COVID-19 screening clinic at the Chosun University Hospital because he returned from an endemic country of COVID-19. He was determined to be positive by RT-PCR of SARS-CoV-2 genes and subsequently hospitalized. At the time of hospitalization, pneumonia was not detected in both chest X-ray (Fig. 1b) and high-resolution computed tomography (HRCT). He complained of a sore throat on the next day after admission, and developed a fever of about $38^{\circ} \mathrm{C}$ on day 1 and day 2 after symptom onset. Hydroxychloroquine was started on day 4 because he began to have a productive cough with fever. Hydroxychloroquine treatment was continued for 5 days whereupon he was stable with conservative treatments without fever or other symptoms.

Chest radiographs were obtained daily using conventional radiography, which was performed at the bedside. Chest radiographic scores were calculated using chest X-rays obtained during hospital stay. The scores were calculated by dividing each lung into upper, middle, and lower zones and scoring of each zone from 0 to 4 points was based on the degree of infiltration. The scores of the six zones are then added, yielding a total score ranging from 0 to $24 .{ }^{28}$

\section{Cytological analysis}

In P1 and P2, bronchoscopy was performed under conscious sedation using intravenous midazolam. Heart rate, respiratory rate, blood pressure, and oxygen saturation were monitored before, during, and after the procedure. BALF was performed with a single-use disposable bronchoscope (Ambu ${ }^{\circledR}$ aScope ${ }^{\mathrm{TM}} 4$, Ambu A/S, Denmark) in a wedged position within a subsegmental bronchus by a pulmonologist. Sterile $0.9 \% \mathrm{NaCl}$ saline was instilled in the selected subsegmental bronchus in sequential aliquots $(30,30$, and $40 \mathrm{~mL}$ ). After saline instillation, the fluid was immediately recovered into the same syringe by gentle and continuous manual aspiration. BAL and sputum cytology samples were obtained and smeared on glass 
slides immediately after collection. The slides were then immediately fixed with $95 \%$ ethanol in a Coplin jar. After fixing the slides, hematoxylin and eosin (H\&E) staining was performed.

\section{Flow cytometry}

Peripheral blood leukocytes and mononuclear cells (PBMCs) were prepared by standard density gradient centrifugation with Histopaque-1077 and 1119 (Sigma-Aldrich, St. Louis, MO, USA) in biosafety level 3 laboratory in Seoul National University. Blood leukocytes and PBMCs were cryopreserved in liquid nitrogen or directly stained with antibodies listed below for flow cytometry in biosafety level 3 laboratory. Dead cells were stained with Zombie Aqua Fixable Viability Dye (BioLegend, San Diego, CA, USA). Cells were stained with the following sets of antibodies differentially labeled with indicated fluorochrome; antiCD4-Alexa488, anti-CD8-PerCP, anti-CD14-BV605, anti-CD16-Alexa700, anti-CD24-BV421, anti-CD45-PerCPCy5.5, anti-CD206-Alexa488, anti-HLA-DR-BV711 (from BioLgend), anti-CD3-PE-CF594 or Pacific blue, antiCD8-PerCP or APC, anti-CD20-APC/H7, and anti-CD56-APC (from BD Bioscience). PE-conjugated CD1d/PBS57 tetramers obtained from the National Institute of Health Tetramer Core Facility (Bethesda, MD, USA) were kindly supplied by Dr. Doo Hyun Cheong at Seoul National University College of Medicine. Cell were then fixed with a fixation buffer (BD Bioscience) and analyzed using a FACS Fortessa II flow cytometer (BD Biosciences). Data were analyzed using Flowjo software (Tree Star, Ashland, OR, USA). Gating strategies for the flow cytometric analysis are summarized in Fig. S1.

\section{Enzyme-linked immunosorbent assay (ELISA)}

To assess for SARS-CoV N protein-specific antibody responses, 96-well immunoassay plates (Nunc, Waltham, MA, USA) were coated with $100 \mu \mathrm{L}$ of purified antigen at a concentration of $1 \mu \mathrm{g} / \mathrm{mL}$ at $4{ }^{\circ} \mathrm{C}$ overnight. The plates were then blocked for $2 \mathrm{~h}$ at room temperature (RT) with PBS containing $5 \%$ skim milk. One hundred microliters of serially diluted plasma samples were incubated for $2 \mathrm{~h}$ at RT. After washing with PBS containing 0.05\% Tween 20 (0.05\% PBST), horseradish peroxidase (HRP)-conjugated mouse anti-human IgG1, IgG2, IgG3, IgG4, IgA or IgE antibody (Southern Biotech, Birmingham, AL, USA) was added and incubated for $1 \mathrm{~h}$ at RT. Wells were washed with $0.05 \%$ PBST and incubated with a 3,3',5,5'-tetramethylbenzidine (TMB) peroxidase substrate solution (KPL, Gaithersburg, MD, USA) for $10 \mathrm{~min}$. The reactions were stopped by addition of a $1 \mathrm{M}$ phosphoric acid solution. Absorbance was measured at $450 \mathrm{~nm}$ using a microplate reader (Beckman Coulter, Brea, CA, USA). The cut-off titer for the ELISA was determined as the lowest titer showing an optical density (OD) over the mean OD plus $3 \times$ standard deviation (s.d.) from three control plasma samples (diluted 1:10).

\section{Quantitation of viral loads}

Real-time reverse transcription-polymerase chain reaction (RT-PCR) assay for detection of SARS-CoV-2 was performed according to the manufacturer's instructions (Kogenebiotech, Seoul, South Korea). Total RNAs were from samples obtained by nasopharyngeal and throat swabs (upper respiratory tract) and sputa (lower respiratory tract). Primer sets targeting E and RdRP genes of SARS-CoV-2 were used with a cut-off cycle threshold (Ct) value of higher than 38 cycles. 


\section{Quantitation of complements}

Human complement assays were conducted using quantitative C3a and C5a ELISA kits according to the manufacturer's instructions (Thermo Fisher Scientific, Waltham, MA, USA). In brief, patients' plasma were incubated in microwells adsorbed with anti-human C3a or C5a coating antibody for $2 \mathrm{~h}$ at RT, washed 6 times, and incubated with a biotin-conjugated anti-human C3a or C5a antibody for $1 \mathrm{~h}$. The plates were then washed 6 times followed by incubation with streptavidin-HRP for $1 \mathrm{~h}$. After washing the wells, TMB substrate solution was added and further incubated at RT. The reaction was terminated by adding $1 \mathrm{M}$ phosphoric acid and absorbance was measured with a TECAN microplate reader (TECAN, Mannedorf, Switzerland) at $450 \mathrm{~nm}$.

\section{Statistical analysis}

Data was analyzed using the Graph Pad Prism 5.01 software (GraphPad Software, La Jolla, CA, USA). Statistical analysis was performed using one-way analysis of variance (ANOVA) followed by NewmanKeuls t-test for comparisons of values among different groups. A $p$-value of $<0.05$ was considered statistically significant.

\section{Declarations}

\section{Acknowledgement}

This research was supported by a grant from the National Research Foundation of Korea (grant no. 2017M3A9E4061998), which are funded by the government of South Korea. We are grateful to Dr. Hyoung-Shik Shin and Prof. Doo Hyun Chung for their advice and comments.

\section{References}

1 Zhu, N. et al. A Novel Coronavirus from Patients with Pneumonia in China, 2019. N Engl J Med 382, 727-733, doi:10.1056/NEJMoa2001017 (2020).

2 Zhou, F. et al. Clinical course and risk factors for mortality of adult inpatients with COVID-19 in Wuhan, China: a retrospective cohort study. Lancet 395, 1054-1062, doi:10.1016/S0140-6736(20)30566-3 (2020).

3 Yang, X. et al. Clinical course and outcomes of critically ill patients with SARS-CoV-2 pneumonia in Wuhan, China: a single-centered, retrospective, observational study. Lancet Respir Med, doi:10.1016/S2213-2600(20)30079-5 (2020).

4 De Giacomi, F., Vassallo, R., Yi, E. S. \& Ryu, J. H. Acute Eosinophilic Pneumonia. Causes, Diagnosis, and Management. Am J Respir Crit Care Med 197, 728-736, doi:10.1164/rccm.201710-1967Cl (2018). 
$5 \quad$ Grp, C. S. The species Severe acute respiratory syndrome-related coronavirus: classifying 2019nCoV and naming it SARS-CoV-2. Nature Microbiology 5, 536-544, doi:10.1038/s41564-020-0695-z (2020).

6 Kim, E. S. et al. Clinical Course and Outcomes of Patients with Severe Acute Respiratory Syndrome Coronavirus 2 Infection: a Preliminary Report of the First 28 Patients from the Korean Cohort Study on COVID-19. J Korean Med Sci 35, e142, doi:10.3346/jkms.2020.35.e142 (2020).

7 Barnig, C. et al. Circulating Human Eosinophils Share a Similar Transcriptional Profile in Asthma and Other Hypereosinophilic Disorders. PLOS ONE 10, e0141740, doi:10.1371/journal.pone.0141740 (2015).

8 Das, R., Sant'Angelo, D. B. \& Nichols, K. E. Transcriptional control of invariant NKT cell development. Immunological reviews 238, 195-215, doi:10.1111/j.1600-065X.2010.00962.x (2010).

9 Yu, Y. R. et al. Flow Cytometric Analysis of Myeloid Cells in Human Blood, Bronchoalveolar Lavage, and Lung Tissues. Am J Respir Cell Mol Bio/ 54, 13-24, doi:10.1165/rcmb.2015-01460C (2016).

10 Godfrey, D. I., MacDonald, H. R., Kronenberg, M., Smyth, M. J. \& Van Kaer, L. NKT cells: what's in a name? Nat Rev Immunol 4, 231-237, doi:10.1038/nri1309 (2004).

11 Bilenki, L., Yang, J., Fan, Y., Wang, S. \& Yang, X. Natural killer T cells contribute to airway eosinophilic inflammation induced by ragweed through enhanced IL-4 and eotaxin production. European journal of immunology 34, 345-354, doi:10.1002/eji.200324303 (2004).

12 Papakosta, D. et al. Bronchoalveolar lavage fluid eosinophils are correlated to natural killer cells in eosinophilic pneumonias. Respiration 78, 177-184, doi:10.1159/000203989 (2009).

13 Qin, C. et al. Dysregulation of immune response in patients with COVID-19 in Wuhan, China. Clin Infect Dis, doi:10.1093/cid/ciaa248 (2020).

14 Pan, Y., Zhang, D., Yang, P., Poon, L. L. M. \& Wang, Q. Viral load of SARS-CoV-2 in clinical samples. Lancet Infect Dis 20, 411-412, doi:10.1016/S1473-3099(20)30113-4 (2020).

$15 \mathrm{Li}, \mathrm{H}$. et al. SAA is a biomarker to distinguish the severity and prognosis of Coronavirus Disease 2019 (COVID-19). J Infect, doi:10.1016/j.jinf.2020.03.035 (2020).

16 Campbell, C. M. \& Kahwash, R. Will Complement Inhibition be the New Target in Treating COVID-19 Related Systemic Thrombosis? Circulation, doi:10.1161/CIRCULATIONAHA.120.047419 (2020).

17 Scott-Taylor, T. H., Axinia, S. C., Amin, S. \& Pettengell, R. Immunoglobulin G; structure and functional implications of different subclass modifications in initiation and resolution of allergy. Immun Inflamm Dis 6, 13-33, doi:10.1002/iid3.192 (2018). 
18 Carr, T. F., Zeki, A. A. \& Kraft, M. Eosinophilic and Noneosinophilic Asthma. Am J Respir Crit Care Med 197, 22-37, doi:10.1164/rccm.201611-2232PP (2018).

19 Dharmage, S. C., Perret, J. L. \& Custovic, A. Epidemiology of Asthma in Children and Adults. Front Pediatr 7, 246, doi:10.3389/fped.2019.00246 (2019).

20 Philit, F. et al. Idiopathic acute eosinophilic pneumonia: a study of 22 patients. Am J Respir Crit Care Med 166, 1235-1239, doi:10.1164/rccm.2112056 (2002).

21 Cao, B. et al. A Trial of Lopinavir-Ritonavir in Adults Hospitalized with Severe Covid-19. N Engl J Med, doi:10.1056/NEJMoa2001282 (2020).

22 Russell, C. D., Millar, J. E. \& Baillie, J. K. Clinical evidence does not support corticosteroid treatment for 2019-nCoV lung injury. Lancet 395, 473-475, doi:10.1016/S0140-6736(20)30317-2 (2020).

23 Wu, C. et al. Risk Factors Associated With Acute Respiratory Distress Syndrome and Death in Patients With Coronavirus Disease 2019 Pneumonia in Wuhan, China. JAMA Intern Med, doi:10.1001/jamainternmed.2020.0994 (2020).

24 Jacobson, K. A., Tosh, D. K., Jain, S. \& Gao, Z. G. Historical and Current Adenosine Receptor Agonists in Preclinical and Clinical Development. Front Cell Neurosci 13, 124, doi:10.3389/fncel.2019.00124 (2019).

25 Heulens, N., Korf, H. \& Janssens, W. Innate immune modulation in chronic obstructive pulmonary disease: moving closer toward vitamin D therapy. J Pharmacol Exp Ther 353, 360-368, doi:10.1124/jpet.115.223032 (2015).

26 Panarese, A. \& Shahini, E. Letter: Covid-19, and vitamin D. Aliment Pharmacol Ther, doi:10.1111/apt.15752 (2020).

27 Chen, L., Xiong, J., Bao, L. \& Shi, Y. Convalescent plasma as a potential therapy for COVID-19. Lancet Infectious Diseases 20, 398-400, doi:10.1016/S1473-3099(20)30141-9 (2020).

28 Conway, S. P. et al. The chest radiograph in cystic fibrosis: a new scoring system compared with the Chrispin-Norman and Brasfield scores. Thorax 49, 860-862, doi:10.1136/thx.49.9.860 (1994).

\section{Tables}


Table 1. Baseline characteristics of three COVID-19 patients included in this study.*

\begin{tabular}{|c|c|c|c|c|c|c|}
\hline \multirow[t]{2}{*}{ ID. } & \multirow[t]{2}{*}{ Age/Sex } & \multirow[t]{2}{*}{ Underlying diseases } & \multirow[t]{2}{*}{ Pneumonia } & \multirow[t]{2}{*}{ Treatment } & \multicolumn{2}{|c|}{ Cytological findings } \\
\hline & & & & & BALF & $\begin{array}{c}\text { sputum, } \\
\text { tracheal aspirate }\end{array}$ \\
\hline P1 & $79 / \mathrm{M}$ & HTN, DM & Severe & $\begin{array}{l}\text { Lopinavir-ritonavir (11 D) } \\
\text { Methylprednisolone (10 D) }\end{array}$ & $\begin{array}{c}\text { Macrophage: } 35 \% \text {, } \\
\text { Lymphocyte: } 20 \% \\
\text { Eosinophil: } 45 \%\end{array}$ & $\begin{array}{c}\text { Macrophage: } 3 \% \\
\text { Eosinophil: } 97 \%\end{array}$ \\
\hline $\mathrm{P} 2$ & $79 / F$ & $\begin{array}{c}\text { HTN, DM } \\
\text { old PTB, GBS }\end{array}$ & Severe & $\begin{array}{l}\text { Lopinavir-ritonavir (10 D) } \\
\text { Methylprednisolone (7 D) }\end{array}$ & $\begin{array}{c}\text { Macrophage: } 45 \% \text {, } \\
\text { Lymphocyte: } 20 \% \\
\text { Eosinophil: } 35 \%\end{array}$ & $\begin{array}{c}\text { Macrophage: } 5 \% \\
\text { Lymphocyte: } 5 \% \\
\text { Eosinophil: } 90 \%\end{array}$ \\
\hline P3 & $36 / \mathrm{M}$ & - & - & $\begin{array}{l}\text { Hydroxychroroquine } \\
\text { (5 D) }\end{array}$ & $\mathrm{N} / \mathrm{A}$ & $\begin{array}{c}\text { Macrophage: } 3 \% \\
\text { Lymphocyte: } 5 \% \\
\text { Eosinophil: } 90 \%\end{array}$ \\
\hline
\end{tabular}

* HTN: Hypertension, DM: Diabetes mellitus, PTB: Pulmonary tuberculosis, GBS: Guillain barre syndrome, BALF: Bronchoalveolar lavage fluid, N/A: not available.

\section{Supplementary Figures}

Fig. S1. Gating strategy for the characterization of leukocytes in blood and BALFs.

Fig. S2. Characterization of lymphocytes in PBMCs from three COVID-19 patients. a. Relative levels of leukocytes' makers of B (brown), NKT-like (red), and T cells (blue) in blood and BALFs from the indicated severe COVID-19 patients. These cell types are gated population from Fig. 2. b. Relative frequencies of $\mathrm{CD}^{+} \mathrm{T}$ cells, $\mathrm{CD} 20^{+} \mathrm{B}$ cells, $\mathrm{CD} 4^{+} \mathrm{T}$ cells, and $\mathrm{CD} 8^{+} \mathrm{T}$ cells among lymphocyte in blood from the indicated patients were arranged based on the collection periods, acute phase (A) and convalescent phase (C). The relative frequencies of each immune cell type from three healthy volunteers were included as controls $(H$, open circles). Black bars indicate the mean value.

Fig. S3. Kinetics of complete blood counts in blood collected from the three COVID-19 patients.

\section{Figures}


a Days after symptom onset

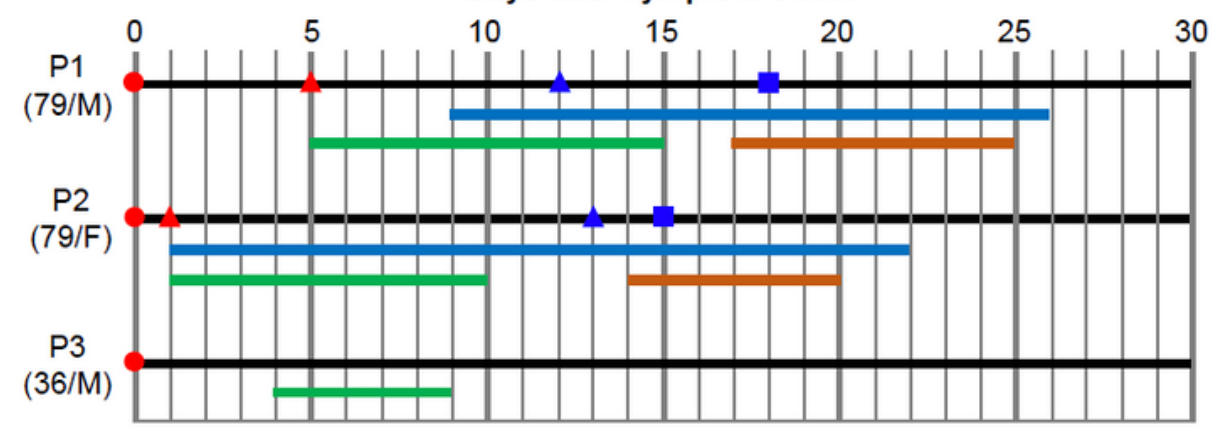

•: Symptom onset $\boldsymbol{\Delta}$ :Pneumonia observed $\boldsymbol{\Delta}$ : Bronchoscopy $\mathbf{\square}$ : Tracheostomy

- Mechanical ventilation $=$ : Anti-viral therapy $=$ : Steroid therapy

b

$\bar{\Sigma}$

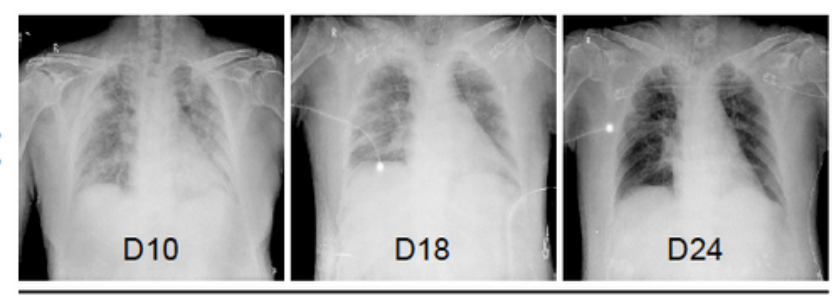

ญ

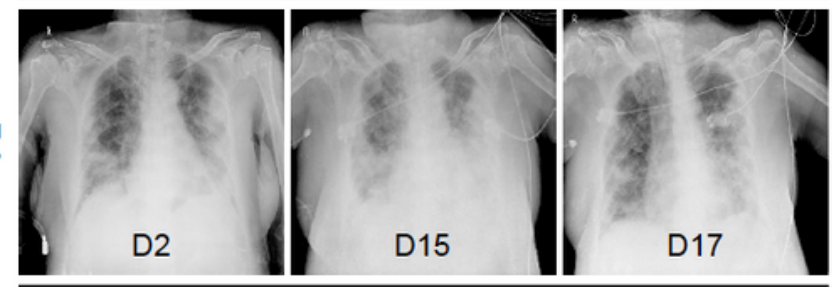

œ
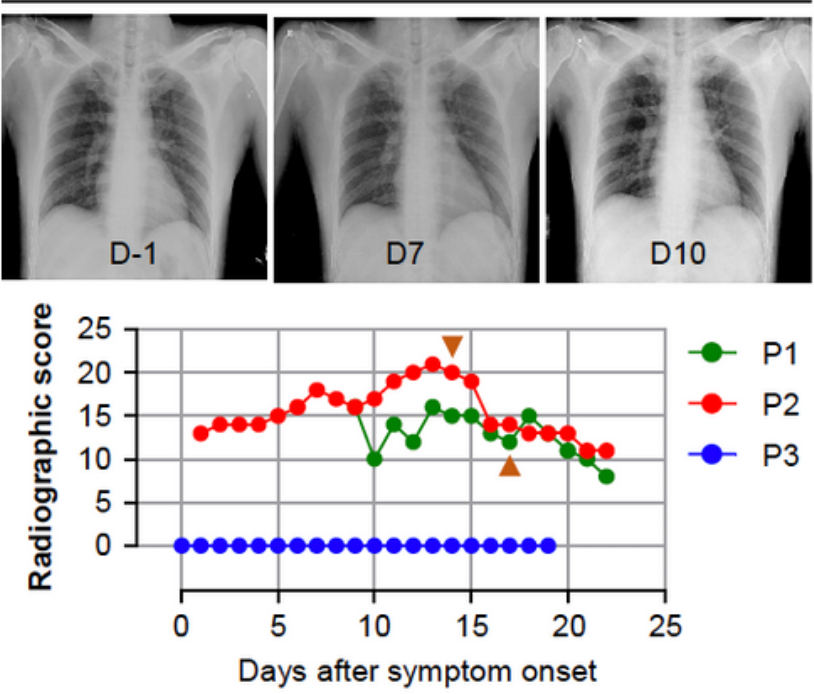

C

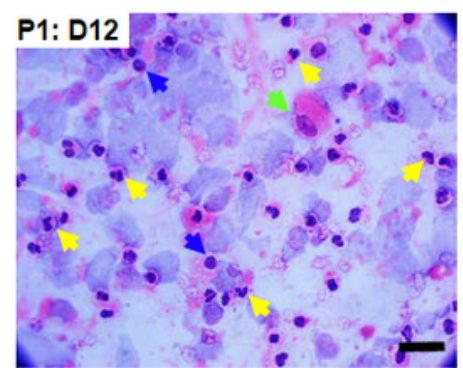

P2: D2

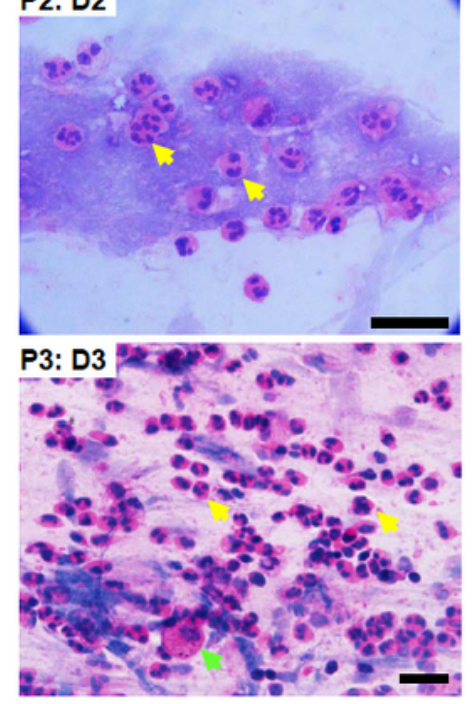

\section{Figure 1}

Clinical courses of three COVID-19 patients included in this study. a. Schematic diagram of clinical courses of the patients and therapies applied. b. Representative chest images (upper images) and their radiographic scores (lower graph) of the COVID-19 patients. Images were taken at the indicated days after symptom onset. Brown arrowheads: commencing day of steroid therapy. c. Representative images of cytological analysis of BALF (P1, upper) and sputa (P2, middle; P3, lower) by H\&E staining. Images were 
taken at the indicated days after symptom onset. Arrows indicate eosinophils (yellow), lymphocytes (blue), and macrophages (green). Scale bar: $20 \mu \mathrm{m}$.

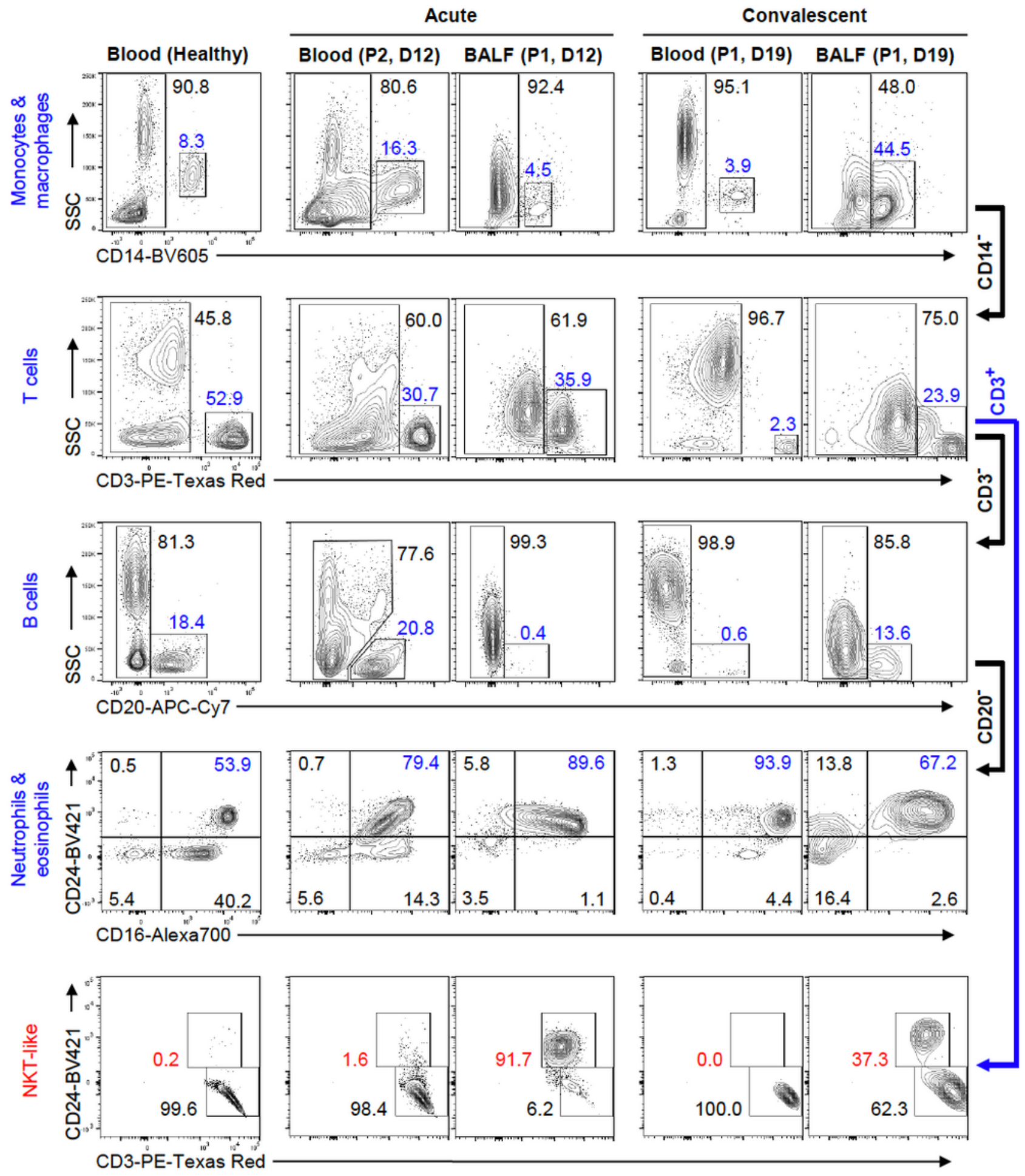

\section{Figure 2}

Relative frequencies of leukocytes subtypes in bloods' and BALFs' leukocytes from severe COVID-19 patients. Flow cytometric analysis of leukocytes in bloods and BALFs collected from two severe COVID19 patients ( $\mathrm{P} 1$ and $\mathrm{P} 2$ ) during acute and convalescent phases were performed after staining with 
indicated antibodies (see Supplementary Fig. 1 for gating strategies). The patients' ID and the sample collection day (days after symptom onset) are indicated in the top. Blood leukocytes collected from a healthy volunteer were used as a control for flow cytometry (left panels). The relative frequencies of specific population were indicated in each window.
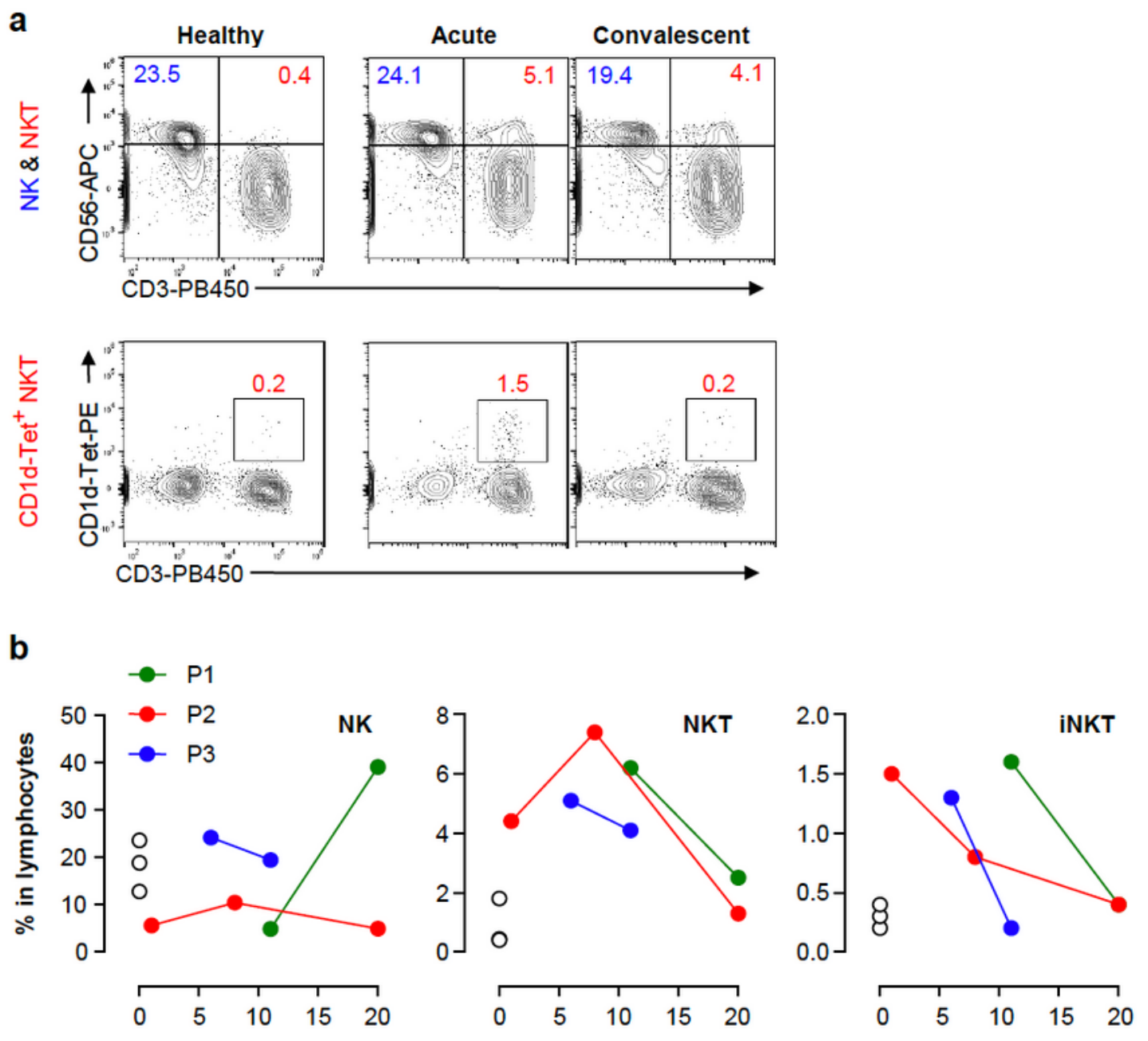

Days after symptom onset
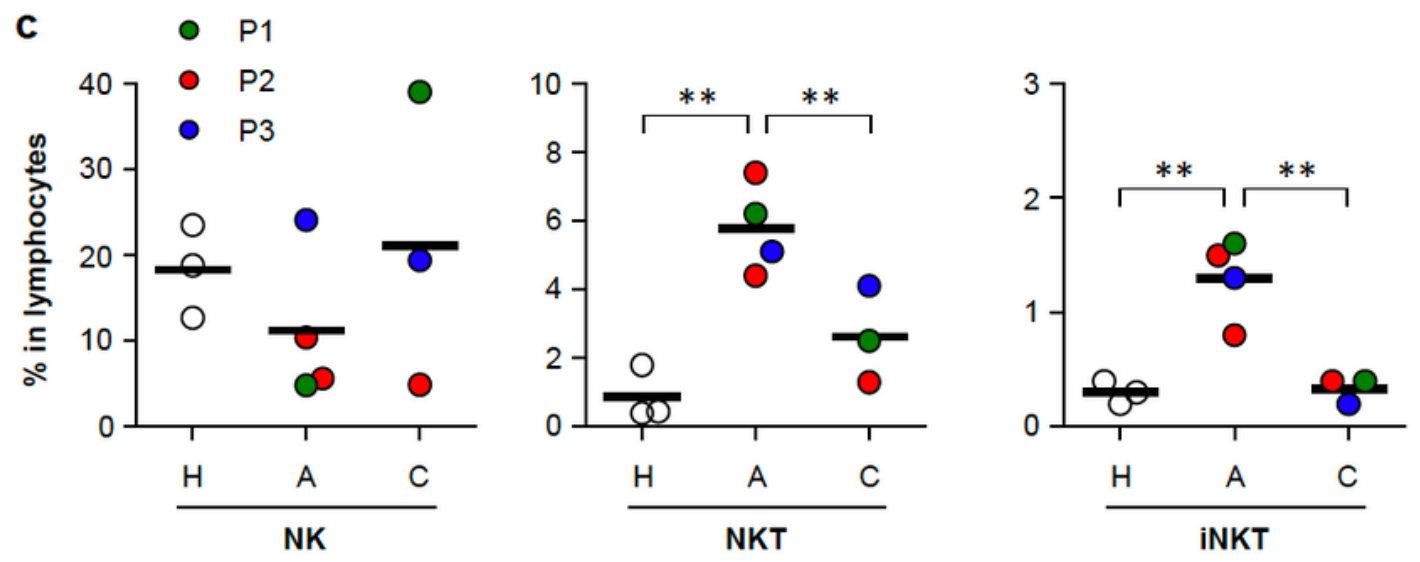

Figure 3 
Relative frequencies of NK and NKT cells among lymphocyte in blood from COVID-19 patients during acute and convalescent phase. a. Representative contour plots identifying NK, NKT, and CD1d-tetramer (Tet)-positive NKT (iNKT) cells using PBMCs from the COVID-19 patients. b. Kinetic changes in the relative frequencies of CD3-/CD56+ NK, CD3+/CD56- NKT, and CD3+/CD1d-tetramer+ iNKT cells among lymphocytes from the indicated patients were presented. The relative frequencies of each immune cell type from three healthy volunteers were included as controls (open circles). c. The relative frequencies of CD3-/CD 56+ NK, CD3+/CD56- NKT, and CD3+/CD1d-tetramer+ iNKT cells among lymphocytes from the indicated patients were rearranged based on the collection periods, acute phase $(A)$ and convalescent phase (C), and compared by one-way analysis of variance (ANOVA) followed by Newman-Keuls t-test. The relative frequencies of each immune cell type from three healthy volunteers were included as controls $(\mathrm{H}$, open circles). Black bars indicate the mean value and $p$ values were obtained using ANOVA followed by Newman-Keuls t-test. **: $p<0.01$. 
a
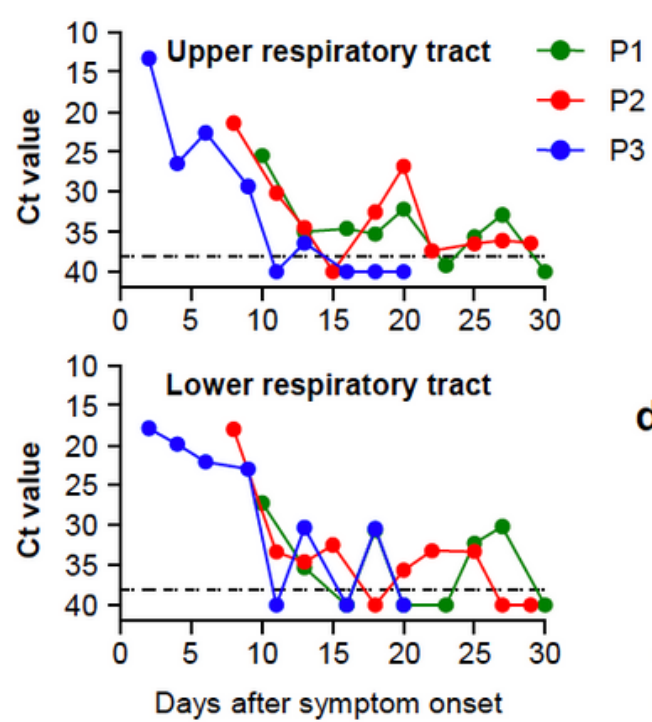

b

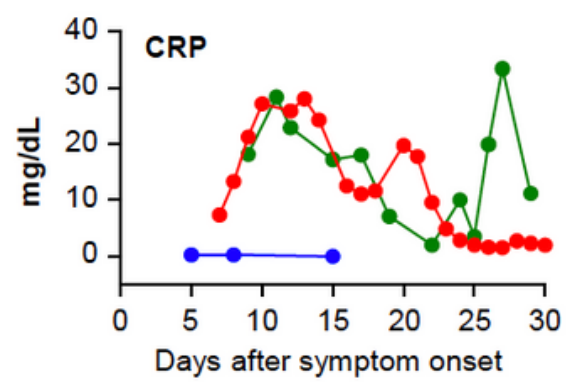

C

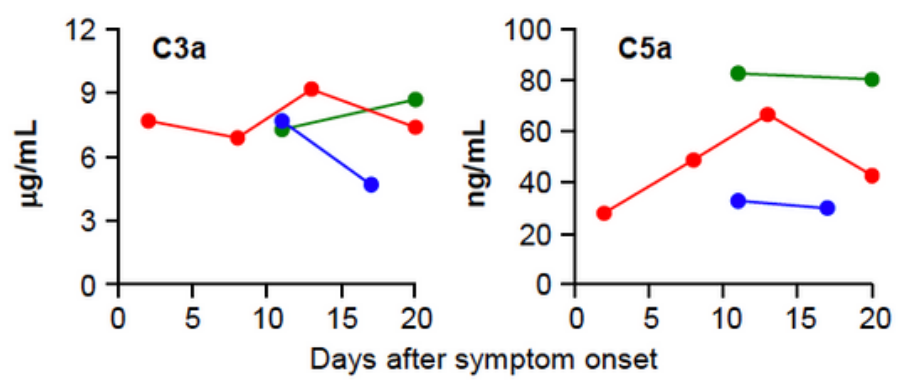

d

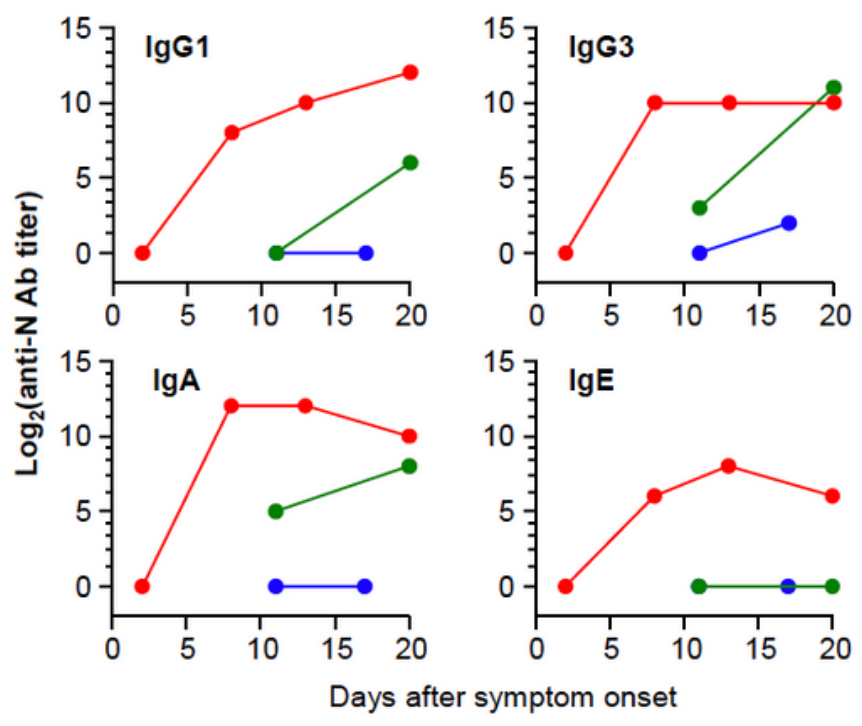

e

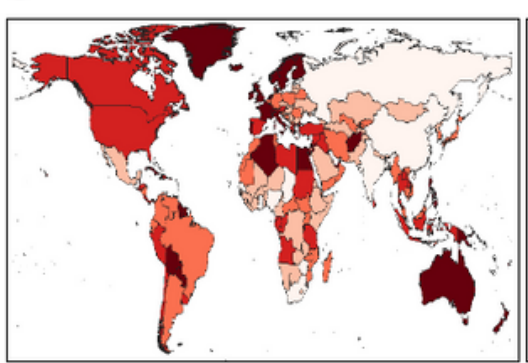

Asthma (cases $/ 100,000$ )

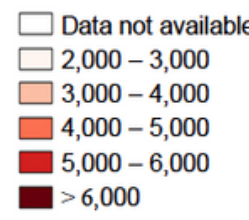

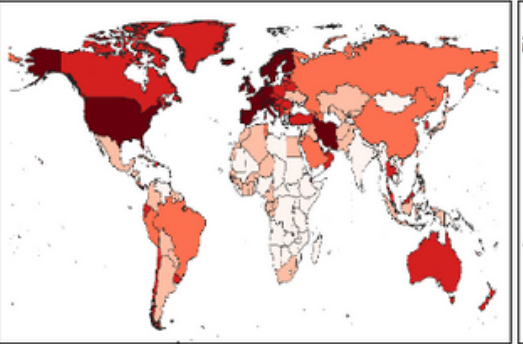

COVID-19 (cases $/ 100,000$ )

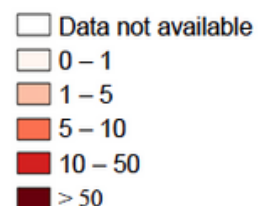

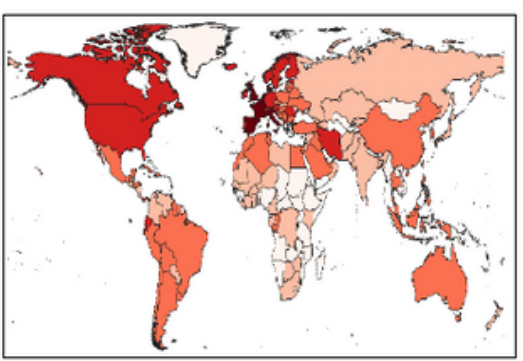

COVID-19 (deaths $/ 100,000$ )

$\square$ Data not available

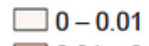

$\square 0.01-0.1$

$\square 0.1-1$

$\square 1-10$

r $>10$

\section{Figure 4}

Kinetic changes of respiratory viral loads and levels of C-reactive protein, complements, and SARS-CoV-2 $\mathrm{N}$-specific antibodies of the indicated isotype in plasma. a. Kinetic changes of viral loads were evaluated in respiratory specimens (nasopharygeal/throat swabs for upper respiratory tract and sputa for lower respiratory tract) by quantitative RT-PCR targeting E gene of SARS-CoV-2 and presented as Ct values. Dotted line: cut-off value. b - d. Kinetic changes of C-reactive proteins (CRP), complements (C3a and C5a), 
and specific antibodies against viral $\mathrm{N}$ protein are presented. Specific isotypes of the antigen-specific antibodies are indicated (d). e. Disease incidence of confirmed COVID-19 and its mortality (World Health Organization, https://covid19.who.int, as of April. 10. 2020) is compared with prevalence of clinical asthma in a global level (Global burden of asthma 2017, Institute for Health Metrics and Evaluation, Seattle, USA). Note: The designations employed and the presentation of the material on this map do not imply the expression of any opinion whatsoever on the part of Research Square concerning the legal status of any country, territory, city or area or of its authorities, or concerning the delimitation of its frontiers or boundaries. This map has been provided by the authors.

\section{Supplementary Files}

This is a list of supplementary files associated with this preprint. Click to download.

- Supplementary.pdf 